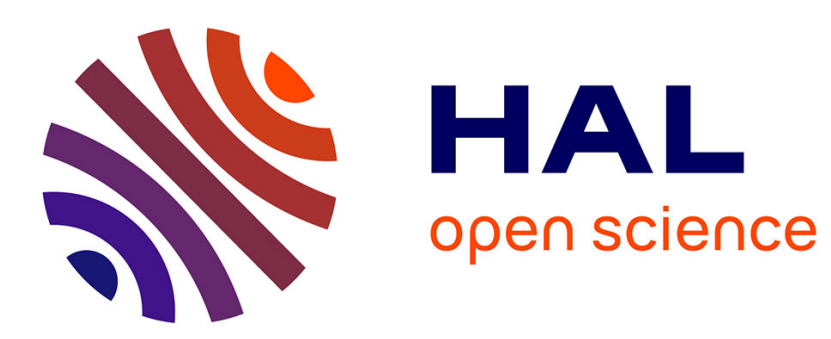

\title{
A feasible direction interior point algorithm for nonlinear semidefinite programming
}

Miguel Aroztegui, Jose Herskovits, Jean Rodolphe Roche

\section{To cite this version:}

Miguel Aroztegui, Jose Herskovits, Jean Rodolphe Roche. A feasible direction interior point algorithm for nonlinear semidefinite programming. 2012. hal-00758803

\section{HAL Id: hal-00758803 \\ https://hal.science/hal-00758803}

Preprint submitted on 29 Nov 2012

HAL is a multi-disciplinary open access archive for the deposit and dissemination of scientific research documents, whether they are published or not. The documents may come from teaching and research institutions in France or abroad, or from public or private research centers.
L'archive ouverte pluridisciplinaire HAL, est destinée au dépôt et à la diffusion de documents scientifiques de niveau recherche, publiés ou non, émanant des établissements d'enseignement et de recherche français ou étrangers, des laboratoires publics ou privés. 


\title{
A feasible direction interior point algorithm for nonlinear semidefinite programming
}

\author{
Miguel Aroztegui · José Herskovits · Jean \\ Rodolphe Roche
}

the date of receipt and acceptance should be inserted later

\begin{abstract}
We present a new algorithm for nonlinear semidefinite programming. It is based on the iterative solution, in the primal and dual variables, of KarushKuhn-Tucker first order optimality conditions. This method generates a decreasing feasible sequence. At each iteration, two linear systems with the same coefficient matrix are solved and an inexact line search is then performed. A proof of global convergence is given in the convex case. Some numerical tests involving nonlinear programming problems as well linear and nonlinear matrix inequalities are described. We also solve structural topology optimization problems employing a mathematical model based on semidefinite programming. The results suggest efficiency and high robustness of the proposed method.
\end{abstract}

Keywords nonlinear, semidefinite programming, feasible directions, interiorpoint methods.

\section{Introduction}

This paper proposes a new technique to solve the following non-linear semidefinite programming problem (SDP),

$$
\begin{aligned}
& \min _{x \in} f(x) \\
& \text { s.t. } G(x) \preccurlyeq 0
\end{aligned}
$$

\footnotetext{
Miguel Aroztegui

Departament of Informatics, Federal University of Paraíba, João Pessoa - Paraíba, CEP: 58.051-900, CT, Cidade Universitária, João Pessoa, Paraíba, Brazil.

E-mail: marozteg@di.ufpb.br

José Herskovits

Mechanical Engineering Program - COPPE - Federal University of Rio de Janeiro, PO Box 68503, CEP 21945-970, CT, Cidade Universitária, Ilha do Fundão, Rio de Janeiro, Brazil.

E-mail: jose@optimize.ufrj.br

Jean R. Roche

I.E.C.N., Université de Lorraine, CNRS, INRIA B.P. 70239, 54506 Vandoeuvre lès Nancy, France.

E-mail: jean-rodolphe.roche@univ-lorraine.fr
} 
where $f: \mathbb{R}^{n} \rightarrow \mathbb{R}$ and $G: \mathbb{R}^{n} \rightarrow \mathbb{S}^{m}$ are smooth functions. We denote $\mathbb{S}^{m}$ the set of real symmetric matrices of size $m \times m$. The constraint $G(x) \preccurlyeq 0$ is a matrix inequality which means that $G(x)$ is negative semidefinite. We call $\Omega=\{x \in$ $\left.\mathbb{R}^{n} ; G(x) \preccurlyeq 0\right\}$ the set of feasible solutions and $\operatorname{int}(\Omega)$, its interior.

Applications in a wide range of areas lead to semidefinite programming problems. For example, combinatorial optimization [3], nonconvex quadratic programming [16], eigenvalue optimization [25], systems control theory [12], matrix completion problems [28], problems in statistics [15] and structural design [2]. In particular SDP was considered to solve free material problems in structural mechanical design, see for example [37], [24], [35], [34] and [38]. Some of this problems are considered in our numerical examples.

When $f$ is linear and $G$ depends affinely on the variables, problem (1) is convex and many efficient algorithms were developed. Duality theory and the central path concept were extended from linear programming to semidefinite programming. Nesterov and Nemirovsky [8] and Alizadeh [3] introduce interior point techniques based on path-following and potential-reduction approaches. In Todd, [36], can be found a deep historical overview.

On the other hand, when $f$ or $G$ are nonlinear functions, problem (1) is called a Nonlinear Semidefinite Programing problem (NSDP). In general, a NSDP problem is nonconvex. Nowadays, there are several methods for NSDP problems. We mention the extension of a primal predictor-corrector interior point method of Jarre [21], the sequentially linear SDP method of Correa and Ramirez [13], which is a generalization of the method presented by Fares, Noll and Apkarian [14] and the augmented Lagrangian approach of Kočvara and Stingl [33]. Also Kanzow, Nagel, Kato and Fukushima [22] presented a successive linearization method with a trust region-type globalization.

A crucial result for semidefinite programming is the characterization of Karush - Kuhn - Tucker (KKT) optimality conditions presented by Shapiro, [31] and [32].

In this paper, we present an interior point algorithm which extends to semidefinite programming the Feasible Direction Interior Point Algorithm, FDIPA. FDIPA is a general technique for smooth nonlinear inequality and equality constrained optimization [17] [18] [29] [19]. The present algorithm, defines first at each interior point a feasible and descent direction with respect to the semidefinite constraints. Then, makes a line search in that direction to obtain a new interior point with a lower objective. A Newton or quasi - Newton and first order versions of presented algorithm can be obtained. The present method is simple to code, does not require the solution of quadratic programs and it is not a penalty neither a barrier method. It merely requires the solution of two linear systems with the same matrix per iteration.

The paper is organized as follows. In sub-section 1.1 we introduce some notations and basic concepts about symmetric matrices, the symmetric Kronecker product and the Lagrangian of the problem. In sub-section 1.2 we present some definitions and technical results. Following [31] and [32] we recall first order optimality conditions adapted to semi-definite programming. In section 2 we describe the main ideas of the algorithm. We show how the search direction is built to give a feasible and descent direction. At the end of this section we introduce the general statement of the presented algorithm. The global convergence to a critical point of the considered optimization method is proved in section 3. Details of the imple- 
mentation of the presented algorithm are shown in section 4. Numerical examples are presented in section 6 . The last section is dedicated to conclusions.

\subsection{Notation}

We introduce some notation concerning the matrix spaces. Let $\mathbb{R}^{m \times n}$ denote the space of $m \times n$ real matrices. Let $\mathbb{S}^{n}$ denote the space of real symmetric matrices. The set of symmetric positive semidefinite and positive definite matrices of size $m \times m$ are denoted $\mathbb{S}_{+}^{m}$ and $\mathbb{S}_{++}^{m}$, respectively. In an analog way the set of negative semidefinite and negative definite matrix of size $m$ are denoted $\mathbb{S}_{-}^{m}$ and $\mathbb{S}_{--}^{m}$, respectively. The symbol $\preccurlyeq$ defines the negative semidefinite order, that is, $A \preccurlyeq B$ means that $A-B$ is negative semidefinite. Similarly, the symbol $\prec, \succcurlyeq$ and $\succ$ defines the negative definite, positive semidefinite and positive definite order, respectively.

The symmetric part of $M \in \mathbb{R}^{n \times n}$ is indicated by $\operatorname{sym}(M)$ and the skew part as $\operatorname{skw}(M)$. We denote $\operatorname{ker}(A)$ as the null space of the matrix $A \in \mathbb{R}^{m \times n}$.

The $(i, j)$ th entry of a matrix $A \in \mathbb{R}^{m \times n}$ is referred to by $a_{i j}$. The transpose of $A$ is written as $A^{\top}$. Let $I_{n}$ denote the identity matrix in $\mathbb{R}^{n \times n}$.

Given $A \in \mathbb{S}^{m}$, then $\bar{m}=\frac{1}{2} m(m+1)$ is the number of upper diagonal components of $A$. To introduce the symmetric Kronecker product, we define the two following maps:

$$
\begin{aligned}
& \text { svec }: \mathbb{S}^{m} \rightarrow \mathbb{R}^{\bar{m}}
\end{aligned}
$$

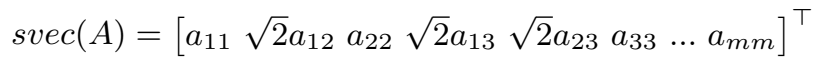

and smat $: \mathbb{R}^{\bar{m}} \rightarrow \mathbb{S}^{m}$ the inverse of svec, see [23].

Then, the inner product

$$
\langle A, B\rangle=\operatorname{tr}\left(A^{\top} B\right)=\operatorname{svec}(A)^{\top} \operatorname{svec}(B), \text { for } A, B \in \mathbb{S}^{m} .
$$

The symmetric Kronecker product of two matrices $A, B \in \mathbb{R}^{m \times n}$ is represented by $A \circledast B$ and verifies for any matrix $C \in \mathbb{S}^{n}$ the following equality:

$$
(A \circledast B) \operatorname{svec}(C)=\operatorname{svec}(\operatorname{sym}(B C A))
$$

It is proved that, [23]:

1. $(A \circledast B) \operatorname{svec}(C)=(B \circledast C) \operatorname{svec}(A)$

2. $A \circledast B=B \circledast A$

3. $(A \circledast B)(C \circledast D)=\frac{1}{2}(A C \circledast B D+A D \circledast B C)$

4. $(P \circledast P)^{-1}=P^{-1} \circledast P^{-1}$

5. If $A, B \succcurlyeq 0$ then, $A \circledast B \succcurlyeq 0$.

6. If $A \succcurlyeq 0$ and $B \preccurlyeq 0$ then, $A \circledast B \preccurlyeq 0$.

where $A, B, C \in \mathbb{S}^{m}$ and $P$ is a non singular matrix.

The partial derivatives of $G(x)$ with respect to $x_{p}$ is denoted by $\frac{\partial G}{\partial x_{p}}(x)$, with components $\frac{\partial g_{i j}(x)}{\partial x_{p}}, i, j=1, m$. 
Then, we can define the following matrix in $\mathbb{R}^{n \times \bar{m}}$,

$$
\nabla G(x)=\left[\begin{array}{c}
\operatorname{svec}\left(\frac{\partial G}{\partial x_{1}}(x)\right)^{\top} \\
\vdots \\
\operatorname{svec}\left(\frac{\partial G}{\partial x_{n}}(x)\right)^{\top}
\end{array}\right] .
$$

The derivative of $G$ in the direction $d \in \mathbb{R}^{n}$ at $x$, denoted by $D G(x) d$, verifies:

$$
D G(x) d=\sum_{p=1}^{n} d_{p} \frac{\partial G}{\partial x_{p}}(x)
$$

In view of (4), the matrix equation (5) can be expressed in a vector format

$$
\operatorname{svec}(D G(x) d)=\nabla G(x)^{\top} d
$$

The Lagrangian function of problem (1) is defined as $L: \mathbb{R}^{n} \times \mathbb{S}^{m} \rightarrow \mathbb{R}^{n}$ such that

$$
L(x, \Lambda)=f(x)+\langle G(x), \Lambda\rangle .
$$

Alternatively, the Lagrangian can be written in the form $L: \mathbb{R}^{n} \times \mathbb{R}^{\bar{m}} \rightarrow \mathbb{R}^{n}$ such that

$$
L(x, \lambda)=f(x)+\langle\operatorname{svec}(G(x)), \lambda\rangle
$$

where $\lambda=\operatorname{svec}(\Lambda)$.

Therefore the gradient of the Lagrangian with respect to $x$ can be written as

$$
\nabla_{x} L(x, \lambda)=\nabla f(x)+\nabla G(x) \lambda,
$$

see [32] and [10].

1.2 Basic definitions and technical results

The following three definitions are related with first order optimality conditions for semidefinite programming analyzed in [31] and [32].

We call $\left\{b_{1}(x), \ldots, b_{p}(x)\right\}$ an orthonormal basis of $\operatorname{ker} G(x)$ and define the matrix $E_{0}(x)=\left[b_{1}(x) \ldots b_{p}(x)\right] \in \mathbb{R}^{m \times p}$.

Definition 1 A point $x$ is a regular point of problem (1) if the vectors of the set

$$
\left\{\left[\begin{array}{c}
b_{i}^{\top} \frac{\partial G}{\partial x_{1}}(x) b_{j} \\
\vdots \\
b_{i}^{\top} \frac{\partial G}{\partial x_{n}}(x) b_{j}
\end{array}\right] \text { such that } i \leqslant j, i, j=1, \ldots, p\right\}
$$

are linearly independent. 
Definition 2 A regular point $x$ is a stationary point of problem (1) if there exist $\Lambda \in \mathbb{S}^{m}$ such that the following requirements are verified:

$$
\begin{aligned}
\nabla_{x} L(x, \Lambda) & =0 \\
\Lambda G(x) & =0 \\
G(x) & \preccurlyeq 0
\end{aligned}
$$

Definition 3 A Karush-Kuhn-Tucker point of problem (1) is a stationary point and $\Lambda \succcurlyeq 0$.

Definition 4 The vector $d \in \mathbb{R}^{n}$ is a feasible direction of $\Omega$ at $x \in \Omega$ if there exists $\tau>0$ such that $x+t d \in \Omega$ when $t \in[0, \tau]$.

Definition 5 The vector field $d(x)$ defined on $\Omega \subseteq \mathbb{R}^{n}$ is said to be an uniformly feasible direction field of $\Omega$ if there exists $\tau>0$ such that, $x+t d(x) \in \Omega$ for all $x \in \Omega$

and $t \in[0, \tau]$.

When the vector field $d(x)$ is a uniformly feasible direction field of $\Omega$, the segment $[x, x+\tau d(x)]$ is included in $\Omega$ for all $x \in \Omega$. When $x$ is in the interior of $\Omega$, all direction are feasible.

Definition $6 d \in \mathbb{R}^{n}$ is a descent direction of a real function $f$ at $x \in \mathbb{R}^{n}$ if there exist some $\delta>0$ such that::

$f(x+t d)<f(x)$ for all $t \in(0, \delta]$.

It is possible to prove that if $f$ is differentiable at $x$ and $d^{\top} \nabla f(x)<0$ then $d$ is a descent direction of $f$ at $x$.

Now we introduce some technical result useful to describe and prove the convergence of the studied algorithm.

Lemma $1 A \in \mathbb{S}_{-}^{m}$ and $B \in \mathbb{S}_{+}^{m}$, the following equalities are equivalent

$$
\begin{aligned}
A B & =0 \\
\operatorname{tr}(A B) & =0 \\
\operatorname{sym}(A B) & =0
\end{aligned}
$$

Proof The proof for $A B=0 \Longleftrightarrow \operatorname{tr}(A B)=0$ can be found in [33].

Here we show $A B=0 \Longleftrightarrow \operatorname{sym}(A B)=0$.

$(\Rightarrow)$ Obvious.

$(\Leftarrow)$ By hypothesis, $\operatorname{sym}(A B)=0$ and since $\operatorname{tr}(\operatorname{skw}(A B))=0$, the trace of $A B$ is null. Since $A \in \mathbb{S}_{-}^{m}$ and $B \in \mathbb{S}_{+}^{m}$, there exist $P, Q \in \mathbb{S}^{m}$ such that $A=-P P$ and $B=Q Q$. Then we have

$$
\begin{aligned}
0 & =\operatorname{tr}(A B)=-\operatorname{tr}(P P Q Q)=-\operatorname{tr}(Q P P Q)= \\
& =-\langle P Q, P Q\rangle=-\|P Q\|^{2} .
\end{aligned}
$$

Then $P Q=0$. As a result, $P P Q Q=0$ and $A B=0$.

Lemma 2 If $A, B \in \mathbb{S}^{m}, A \succ 0$ and $A B+B A \prec 0$ then $B \prec 0$. 
Proof Suppose that there exist an eigenvalue $\lambda \geqslant 0$ of $B$ with an eigenvector $v$. Then,

$$
v^{\top}(A B+B A) v=v^{\top} A B v+v^{\top} B A v=2 \lambda v^{\top} A v
$$

Since $A$ is positive definite, $\lambda v^{\top} A v \geqslant 0$ therefore, $v^{\top}(A B+B A) v \geqslant 0$, and we conclude that $A B+B A$ is not negative definite, which is a contradiction with the hypothesis.

The proof of following lemma is similar as above.

Lemma 3 If $A, B \in \mathbb{S}^{m}, A \succ 0$ and $A B+B A \preccurlyeq 0$ then $B \preccurlyeq 0$.

Lemma 4 If $A, B \in \mathbb{S}^{m}$ and $A \succ 0$, the matrix $A B$ has real eigenvalues and the same inertia as B. See [20].

Lemma 5 If $A \in \mathbb{S}_{++}^{m}$ and $B \in \mathbb{S}_{-}^{m}$ and they commute, then

$$
y^{\top} A B y=0 \Longleftrightarrow B y=0 .
$$

Proof $(\Rightarrow)$ : In view of lemma 4 , all eigenvalues of $A B$ are non positive. Let $\lambda_{1}, \ldots, \lambda_{m}$ and $\left\{b_{1}, \ldots, b_{m}\right\}$ be the eigenvalues and the orthonormal eigenvectors of $A B$, respectively. Let $y$ be a not null vector in $\mathbb{R}^{m}$ such that $y^{\top}(A B) y=0$. Then,

$$
y=\sum_{j=1}^{m} \alpha_{j} b_{j}
$$

and

$$
\begin{aligned}
0 & =y^{\top}(A B) y=\langle y,(A B) y\rangle \\
& =\sum_{j=1}^{m} \sum_{i=1}^{m} \alpha_{i} \alpha_{j} \lambda_{i}\left\langle b_{i}, b_{j}\right\rangle=\sum_{i=1}^{m} \alpha_{i}^{2} \lambda_{i}
\end{aligned}
$$

Since, $y \neq 0$, there exist $\alpha_{i} \neq 0$. In view of the last equation, $\lambda_{i}$ must be null, therefore $y \in \operatorname{ker}(A B)$, but $A \in \mathbb{S}_{++}^{m}$ then $y \in \operatorname{ker}(B)$.

Lemma 6 If $A \in \mathbb{S}_{-}^{m}$ and $B \in \mathbb{S}^{m}$ then,

$$
A B^{2}=0 \Longleftrightarrow \operatorname{sym}(A B)=0
$$

Proof $(\Rightarrow): A \in \mathbb{S}_{-}^{m}$ then there exist $Q \in \mathbb{S}^{m}$ such that $A=-Q Q$.

$0=\operatorname{tr}\left(A B^{2}\right)=\operatorname{tr}(B A B)=-\operatorname{tr}(B Q Q B)=-\|B Q\|^{2}$, then $B Q=0$ and $-B Q Q=$ $B A=0$.

$(\Leftarrow): A B+B A=0 \Leftrightarrow A B=-B A \Rightarrow A B^{2}=-B A B \Leftrightarrow A$ and $B^{2}$ are simultaneously diagonalizable and $A B^{2} \preccurlyeq 0$. On the other hand, $-B A B=B(-A) B \succcurlyeq 0$. Then, $0 \preccurlyeq-B A B=A B^{2} \preccurlyeq 0$. Then, we conclude that $A B^{2}=0$.

Lemma 7 If we assume $A \in \mathbb{S}_{++}^{m}$ and $B \in \mathbb{S}_{-}^{m}$ and they commute then,

$$
(A \circledast I)^{-1}(B \circledast I) \in \mathbb{S}_{-}^{\bar{m}} .
$$


Proof Since $A \succ 0$ and $B \preccurlyeq 0$ and commute then there exist a regular matrix $P \in \mathbb{R}^{m \times m}$ such that

$$
\begin{aligned}
& A=P D_{A} P^{-1} \\
& B=P D_{B} P^{-1}
\end{aligned}
$$

In view of symmetric Kronecker product properties, see for example lemma E.1.2 in $[23]$ :

$$
\begin{aligned}
& A \circledast I=\mathrm{PD}_{A} \mathrm{P}^{-1} \\
& B \circledast I=\mathrm{PD}_{B} \mathrm{P}^{-1}
\end{aligned}
$$

where $\mathrm{P}=P \circledast P, \mathrm{D}_{A}=D_{A} \circledast I$ e $\mathrm{D}_{B}=D_{B} \circledast I . \mathrm{D}_{A}$ e $\mathrm{D}_{B}$ are diagonal matrices and $P$ is orthonormal. Therefore

$$
(A \circledast I)^{-1}(B \circledast I)=\mathrm{PD}_{A} \mathrm{D}_{B} \mathrm{P}^{\top} \in \mathbb{S}_{-}^{\bar{m}}
$$

Lemma 8 Let $B \in \mathbb{S}_{-}^{m}$ and $\left\{b_{1}, \ldots, b_{p}\right\}$ be an orthonormal basis of $\operatorname{ker}(B)$ and $E_{0}=\left[b_{1}, \ldots, b_{p}\right] \in \mathbb{R}^{m \times p}$. The following sets are equal:

$$
\begin{aligned}
& C_{1}=\left\{A \in \mathbb{S}^{m}:\langle A, B\rangle=0, A \succcurlyeq 0, B \preccurlyeq 0\right\} \\
& C_{2}=\left\{E_{0} \theta E_{0}^{\top}: \theta \in \mathbb{S}^{p}, \theta \succcurlyeq 0\right\}
\end{aligned}
$$

Proof First we prove $C_{1} \subset C_{2}$. If $A \in C_{1}, A \succcurlyeq 0$. By hypothesis $B \preccurlyeq 0$ and $\langle A, B\rangle=\operatorname{tr}(A B)=0$. Using proposition $1, A B=0$, consequently $A$ and $B$ are simultaneously diagonalizable, then

$$
A=\left[\begin{array}{ll}
E_{0} & E_{\perp}
\end{array}\right]\left[\begin{array}{cc}
D_{A} & 0 \\
0 & 0
\end{array}\right]\left[\begin{array}{ll}
E_{0} & E_{\perp}
\end{array}\right]^{\top}=E_{0} D_{A} E_{0}^{\top}
$$

where $D_{A} \succcurlyeq 0$ is a diagonal matrix and the columns of $E_{\perp}$ are eigenvectors of $B$ in $\operatorname{ker}(B)^{\perp}$, then, $A \in C_{2}$.

Now we proceed to prove $C_{2} \subset C_{1}$. If $A \in C_{2}, A=E_{0} \theta E_{0}^{\top}$ for some $\theta \succcurlyeq 0$. On the other hand, $\langle A, B\rangle=\operatorname{tr}\left(E_{0} \theta E_{0}^{\top} B\right)=0$ because $E_{0}^{\top} B=0$. Then, $A \in C_{1}$.

\section{Description of the algorithm}

In this section, we introduce the main ideas of the present algorithm.

For a real number $a$, we denote

$$
\Omega_{a}=\{x \in \Omega \text { such that } f(x) \leqslant a\}
$$

and introduce the following assumptions about $f$ and $G$ :

Assumption 1 There exist a real number $a$ such that $\Omega_{a}$ is compact and $\operatorname{int}\left(\Omega_{a}\right) \neq \phi$.

Assumption 2 If $x \in \operatorname{int}\left(\Omega_{a}\right)$ then $G(x) \prec 0$.

Assumption $3 f$ and $G$ are $C^{1}$ in $\Omega_{a}$ and $\nabla f$ and $\frac{\partial G}{\partial x_{p}}$ for $p=1, \ldots, n$ are Lipschitz functions. 
Assumption 4 Any KKT point $x$ is a regular point of problem (1).

The present algorithm makes iterations in the primal and dual variables $(x, \Lambda)$ to solve the equalities in KKT conditions. The method is modified in such a way to satisfy the inequalities at each point. That is, the primal variables are feasible and the dual variables, positive at each iteration.

Many approaches have been proposed to handle the complementarity condition $\Lambda G(x)=0$, see [4] and [36]. The complementarity condition $\Lambda G(x)=0$ is not appropriate for a Newton iteration since the product of two symmetric matrices is not, in general, a symmetric matrix. Instead of $\Lambda G(x)=0$ in our case we consider $\operatorname{sym}(\Lambda G(x))=0$. Since our algorithm generates points $(x, \Lambda)$ such that $G(x) \prec 0$ and $\Lambda \succ 0$, Lemma 1 ensures that $\Lambda G(x)=0$ is equivalent to $\operatorname{sym}(\Lambda G(x))=0$.

Then, the equalities of the stationary point conditions (8) can be rewritten in the following form:

$$
\begin{aligned}
\nabla f(x)+\nabla G(x) \lambda & =0 \\
\operatorname{svec}(\operatorname{sym}(\Lambda G(x))) & =0
\end{aligned}
$$

where $\lambda=\operatorname{svec}(\Lambda)$.

In order to obtain the linear system to be solved at each Newton iteration we define a vectorial function $\psi: \mathbb{R}^{n+\bar{m}} \rightarrow \mathbb{R}^{n+\bar{m}}$, given by the equalities in (14)

$$
\psi(x, \lambda)=\left[\begin{array}{c}
\psi_{l}(x, \lambda) \\
\psi_{c}(x, \lambda)
\end{array}\right]=\left[\begin{array}{c}
\nabla f(x)+\nabla G(x) \lambda \\
\operatorname{svec}(\operatorname{sym}(\Lambda G(x)))
\end{array}\right]
$$

Using the Kronecker product we observe that

$$
\psi_{c}(x, \lambda)=\operatorname{svec}(\operatorname{sym}(I \Lambda G(x))=[I \circledast G(x)] \operatorname{svec}(\Lambda)
$$

and also,

$$
\psi_{c}(x, \lambda)=\operatorname{svec}(\operatorname{sym}(\Lambda G(x) I)=[\Lambda \circledast I] \operatorname{svec}(G(x))
$$

therefore, the Jacobian of $\psi$ is:

$$
\begin{aligned}
& \nabla \psi(x, \lambda)=\left[\begin{array}{cc}
\nabla_{x} \psi_{l}(x, \lambda) & \nabla_{\lambda} \psi_{l}(x, \lambda) \\
\nabla_{x} \psi_{c}(x, \lambda) & \nabla_{\lambda} \psi_{c}(x, \lambda)
\end{array}\right] \\
& =\left[\begin{array}{cc}
\nabla_{x x} L(x, \lambda) & \nabla G(x) \\
(\Lambda \circledast I) \nabla G(x)^{\top} & I \circledast G(x)
\end{array}\right]
\end{aligned}
$$

In a similar way as in [19], instead of computing the Hessian of the Lagrangian $\nabla_{x x} L(x, \lambda)$, we can employ a quasi-Newton matrix or the identity matrix that we denote $B$.

Given a starting point $(x, \Lambda) \in \operatorname{int}\left(\Omega_{a}\right) \times \mathbb{S}_{++}^{m}$, a new estimative $\left(x_{0}, \lambda_{0}\right) \in$ $\mathbb{R}^{n} \times \mathbb{R}^{\bar{m}}$ is obtained by solving the following linear system

$$
\begin{aligned}
{\left[\begin{array}{cc}
B & \nabla G(x) \\
(\Lambda \circledast I) \nabla G(x)^{\top} I \circledast G(x)
\end{array}\right] } & {\left[\begin{array}{c}
x_{0}-x \\
\lambda_{0}-\lambda
\end{array}\right] } \\
& =-\left[\begin{array}{c}
\nabla f(x)+\nabla G(x) \lambda \\
\operatorname{svec}(\operatorname{sym}(\Lambda G(x)))
\end{array}\right]
\end{aligned}
$$

where $\lambda=\operatorname{svec}(\Lambda)$ and $\lambda_{0}=\operatorname{svec}\left(\Lambda_{0}\right)$. We denote $W(x, B, \Lambda)$ the matrix of the system (16).

Calling $d_{0}=x_{0}-x$ we have, 


$$
\begin{aligned}
B d_{0}+\quad \nabla G(x) \lambda_{0} & =-\nabla f(x) \\
(\Lambda \circledast I) \nabla G(x)^{\top} d_{0}+(I \circledast G(x)) \lambda_{0} & =0
\end{aligned}
$$

Note that, if $d_{0}=0$ equation (17) becomes,

$$
\nabla f(x)+\nabla G(x) \lambda_{0}=0
$$

and

$$
(I \circledast G(x)) \lambda_{0}=0
$$

Since $G(x) \prec 0$, the matrix $I \circledast G(x)$ is nonsingular and then, equation (19) makes $\Lambda_{0}=\operatorname{smat}\left(\lambda_{0}\right)=0$. This proves that $\Lambda_{0} G(x)=0$. Now, looking at equation (18) with $\lambda_{0}=0$ we have $\nabla f(x)=0$. Then, $x$ is an stationary point of problem (1).

On the other hand, if $d_{0} \neq 0$, we prove in this paper that $d_{0}$ is a descent direction however we can not ensure that $d_{0}$ is a feasible direction when $x$ is on the boundary of $\Omega$.

To obtain a feasible direction, as in [19], we define another linear system in $(d, \bar{\lambda}) \in \mathbb{R}^{n} \times \mathbb{R}^{\bar{m}}$ with the same matrix and an appropriate negative definite right hand side:

$$
\begin{aligned}
B d+\quad \nabla G(x) \bar{\lambda} & =-\nabla f(x) \\
(\Lambda \circledast I) \nabla G(x)^{\top} d+(I \circledast G(x)) \bar{\lambda} & =-\rho \lambda
\end{aligned}
$$

where $\rho$ is a positive real number and $\lambda=\operatorname{svec}(\Lambda)$. In view of the equation (2) and (6), the second equation of (20) is equivalent to

$$
\operatorname{sym}(\Lambda D G(x)(d)+\bar{\Lambda} G(x))=-\rho \Lambda
$$

where $\bar{\Lambda}=\operatorname{smat}(\bar{\lambda})$.

If $x$ is in the interior of $\Omega$, all direction $d$ is a feasible direction.

In the sequel we introduce the following assumption.

Assumption 5 At every iteration $\Lambda$ and $G(x)$ commute.

Thanks to this last assumption and (21) it will be able to prove that $d$ is a feasible direction for all $x \in \Omega_{a}$.

In view of $(20), d$ converges to $d_{0}$ if $\rho$ goes to zero. Since $d_{0}$ is a descent direction, it verifies $d_{0}^{\top} \nabla f(x)<0$, then imposing an upper bound for $\rho$ such that

$$
d^{\top} \nabla f(x) \leqslant \xi d_{0}^{T} \nabla f(x) \quad \xi \in(0,1)
$$

the feasible direction $d$ is also a descent direction.

The pair $(d, \bar{\lambda})$ obtained by the linear system (20) can also be computed solving

$$
\begin{aligned}
B d_{1}+\quad \nabla G(x) \lambda_{1} & =0 \\
(\Lambda \circledast I) \nabla G(x)^{\top} d_{1}+(I \circledast G(x)) \lambda_{1} & =-\lambda
\end{aligned}
$$

and taking,

$$
\begin{gathered}
d=d_{0}+\rho d_{1} \\
\bar{\lambda}=\lambda_{0}+\rho \lambda_{1} .
\end{gathered}
$$


Imposing (22) to the search direction $d$ defined in (24) we have a precise upper bound for $\rho$. If $d_{1}^{\top} \nabla f(x)>0$, take

$$
\rho \leqslant(\xi-1) \frac{d_{0}^{\top} \nabla f(x)}{d_{1}^{\top} \nabla f(x)} .
$$

Otherwise, we choose

$$
\rho \leqslant \varphi\left\|d_{0}\right\|^{2}
$$

for some fixed parameter $\varphi>0$.

Once we have computed a descent and feasible direction $d$, we can determine the next point in the sequence, $x^{k+1}$, performing a line search along the search direction $d$ to get feasibility and an appropriate reduction of the objective function.

Before starting a new iteration, matrix $B$ can be updated with a new positive definite matrix. Also, matrix $\Lambda$ must be updated with a new positive definite matrix that commutes with $G\left(x^{k+1}\right)$.

2.1 The statement of the algorithm

In this section we state the algorithm under study based on the descent and feasible direction obtained in the previous section.

Input. $x \in \operatorname{int}\left(\Omega_{a}\right)$

Parameters. $\xi \in(0,1), \eta \in(0,1), \varphi>0$ and $\nu \in(0,1)$.

Data. $B \in \mathbb{S}_{++}^{n}, \Lambda \in \mathbb{S}_{++}^{m}$ commuting with $G(x)$.

Step 1. Computation of the search direction $d$.

(i) Solve the following linear system in $d_{0} \in \mathbb{R}^{n}$ and $\lambda_{0} \in \mathbb{R}^{\bar{m}}$

$$
\left[\begin{array}{cc}
B & \nabla G(x) \\
(\Lambda \circledast I) \nabla G(x)^{\top} & I \circledast G(x)
\end{array}\right]\left[\begin{array}{c}
d_{0} \\
\lambda_{0}
\end{array}\right]=\left[\begin{array}{c}
-\nabla f(x) \\
0
\end{array}\right]
$$

If $d_{0}=0$, stop.

(ii) Solve the following linear system in $d_{1} \in \mathbb{R}^{n}$ and $\lambda_{1} \in \mathbb{R}^{\bar{m}}$

$$
\left[\begin{array}{cc}
B & \nabla G(x) \\
(\Lambda \circledast I) \nabla G(x)^{\top} & I \circledast G(x)
\end{array}\right]\left[\begin{array}{c}
d_{1} \\
\lambda_{1}
\end{array}\right]=\left[\begin{array}{c}
0 \\
-\lambda
\end{array}\right]
$$

(iii) Compute the parameter $\rho$ such that

$$
\rho=\min \left\{\varphi\left\|d_{0}\right\|^{2},(\xi-1) \frac{d_{0}^{\top} \nabla f(x)}{d_{1}^{\top} \nabla f(x)}\right\}
$$

if $d_{1}^{\top} \nabla f(x)>0$. Otherwise:

$$
\rho=\varphi\left\|d_{0}\right\|^{2} .
$$

(iv) Compute the search direction $d$ as

$$
d=d_{0}+\rho d_{1} .
$$

Step 2. Line Search.

Find $t$, the first element of $\left\{1, v, v^{2}, v^{3} \ldots\right\}$ such that

$$
f(x+t d) \leqslant f(x)+t \eta d^{\top} \nabla f(x)
$$


and

$$
G(x+t d) \prec 0 .
$$

Step 3. Updates.

(i) Take the new point $x:=x+t d$.

(ii) Define new value for $B \in \mathbb{S}_{++}^{n}$.

(iii) Define new value for $\Lambda \in \mathbb{S}_{++}^{m}$ commuting with $G(x)$.

(iv) Go to Step 1

In the previous algorithm we employ Armijo's line search adapted to constraint optimization problems. Extensions of Wolfe or Goldstein line search criteria [27] could be also employed in step 2 .

We must consider additional assumption on $B$ and $\Lambda$ defined by the previous algorithm in order to prove global convergence.

Assumption 6 There exist positive numbers $\lambda^{I}$ and $\lambda^{S}$ such that

$$
\lambda^{I} I \preccurlyeq \Lambda \preccurlyeq \lambda^{S} I
$$

Assumption 7 There exist positive numbers $\sigma_{1}$ and $\sigma_{2}$ such that

$$
\sigma_{1} I \preccurlyeq B \preccurlyeq \sigma_{2} I
$$

\section{Global convergence}

In this section we prove for any initial point $x^{0} \in \operatorname{int}(\Omega)$, the present algorithm generates a sequence of points converging to a stationary point of problem (1). First we prove that the algorithm is well defined and in particular that the matrix $W(x, B, \Lambda)$ given in (16) is nonsingular. At each iteration $d_{0}$ and $d$ are descent directions of $f$ at $x$ and $d(x)$ is an uniformly feasible directions field in $\Omega_{a}$. Finally we state that any sequence generated by the algorithm converges to a stationary point of (1).

Theorem 1 Assume that $x \in \Omega_{a}$ is a regular point of problem (1), $B \in \mathbb{S}_{++}^{n}$, $\Lambda \in \mathbb{S}_{++}^{m}$ and $\Lambda$ and $G(x)$ commute. Then, the matrix $W(x, B, \Lambda)$ defined in (16) is nonsingular.

Proof We have to prove that, if for some $v \in \mathbb{R}^{n+\bar{m}}$ it is $W(x, B, \Lambda) v=0$, then $v=0$. Let $v^{\top}=\left[r^{\top}, y^{\top}\right], r \in \mathbb{R}^{n}, y \in \mathbb{R}^{\bar{m}}$, then

$$
\begin{gathered}
r=-B^{-1} \nabla G(x) y \\
M y=0
\end{gathered}
$$

where

$$
M=\nabla G(x)^{\top} B^{-1} \nabla G(x)-(\Lambda \circledast I)^{-1}(G(x) \circledast I) .
$$

To finish the proof, we must conclude that $M$ is nonsingular. 
Performing the product $y^{\top} M y$ we have

$$
\begin{gathered}
y^{\top} M y=y^{\top}\left(\nabla G(x)^{\top} B^{-1} \nabla G(x)\right) y \\
-y^{\top}(\Lambda \circledast I)^{-1}(G(x) \circledast I) y
\end{gathered}
$$

Since $B^{-1}$ is positive definite,

$$
y^{\top}\left(\nabla G(x)^{\top} B^{-1} \nabla G(x)\right) y \geqslant 0
$$

By hypothesis and lemma 7

$$
-y^{\top}(\Lambda \circledast I)^{-1}(G(x) \circledast I) y \geqslant 0
$$

concluding that $y^{\top} M y \geqslant 0$.

Now, suppose that $y^{\top} M y=0$. We must prove that $y=0$. From (33), (34) and (35) we have

$$
\begin{aligned}
0 & \leqslant y^{\top}\left(\nabla G(x)^{\top} B^{-1} \nabla G(x)\right) y \\
& =y^{\top}(\Lambda \circledast I)^{-1}(G(x) \circledast I) y \leqslant 0
\end{aligned}
$$

Then,

$$
y^{\top} \nabla G(x)^{\top} B^{-1} \nabla G(x) y=0
$$

and

$$
y^{\top}(\Lambda \circledast I)^{-1}(G(x) \circledast I) y=0 .
$$

Since $B$ is positive definite, from equation (37) we have

$$
\nabla G(x) y=0 .
$$

Due to lemma 5 and from equation (38),

$$
(G(x) \circledast I) y=0 .
$$

By definition of $\nabla G(x)$, equation (39) is equivalent to

$$
\left\langle\frac{\partial G}{\partial x_{i}}(x), Y\right\rangle=0, \quad i=1, \ldots, n
$$

where $Y=\operatorname{smat}(y)$.

Since $(G(x) \circledast I) y=\operatorname{svec}(\operatorname{sym}(G(x) Y))$, in view of lemma 6 and proposition 1 , equation $(40)$ can be rewritten as

$$
\left\langle Y^{2}, G(x)\right\rangle=0
$$

The matrix $Y^{2}$ is positive semidefinite and matrix $G(x)$ is negative semidefinite, then applying lemma 8 we have

$$
Y^{2}=E_{0} \theta E_{0}^{\top}, \theta \in \mathbb{S}^{p}, \theta \succcurlyeq 0
$$

where $E_{0}=\left[b_{1}, \ldots, b_{p}\right] \in \mathbb{R}^{m \times p}$ and the set of vectors $\left\{b_{1}, \ldots, b_{p}\right\}$ is an orthonormal base of $\operatorname{ker}(G(x))$. Taking the square root of $Y^{2}$ in (43), we obtain

$$
Y=E_{0} \theta^{1 / 2} E_{0}^{\top}, \theta^{1 / 2} \in \mathbb{S}^{p}
$$


Replacing $Y$ by $E_{0} \theta^{1 / 2} E_{0}^{\top}$ in equation (41), follows

$$
\left\langle\frac{\partial G}{\partial x_{i}}(x), E_{0} \theta^{1 / 2} E_{0}^{\top}\right\rangle=0, \quad i=1, \ldots, n
$$

which is equivalent to

$$
\left\langle E_{0}^{\top} \frac{\partial G}{\partial x_{i}}(x) E_{0}, \theta^{1 / 2}\right\rangle=0, \quad i=1, \ldots, n
$$

and also equivalent to the following linear system,

$$
V \operatorname{svec}\left(\theta^{1 / 2}\right)=0
$$

where

$$
V=\left[\begin{array}{c}
{\left[\operatorname{svec}\left(E_{0}^{\top} \frac{\partial G}{\partial x_{1}}(x) E_{0}\right)\right]^{\top}} \\
\vdots \\
{\left[\operatorname{svec}\left(E_{0}^{\top} \frac{\partial G}{\partial x_{n}}(x) E_{0}\right)\right]^{\top}}
\end{array}\right] .
$$

We identify in the columns of matrix $V$, vectors of the form

$$
c\left[b_{i}^{\top} \frac{\partial G}{\partial x_{i}}(x) b_{j} \ldots b_{i}^{\top} \frac{\partial G}{\partial x_{n}}(x) b_{j}\right]^{\top}
$$

with $c=\sqrt{2}$ when $i=j$ and $c=1$ when $i \neq j$ where $i, j=1, \ldots, p$ and $i \leqslant j$. By hypothesis, $x$ is a regular point of problem (1), then the columns of $V$ are linearly independent. Then, the linear system (45) has the unique solution $\operatorname{svec}\left(\theta^{1 / 2}\right)=0$. Consequently, $Y=\operatorname{smat}(y)=E_{0} \theta^{1 / 2} E_{0}^{\top}=0$.

As an additional result of the proof of theorem 1, we have that $M$ is positive definite.

Since $\Omega_{a}, \Lambda$ and $B$ are bounded, it follows from theorem 1 that $d_{0}, \lambda_{0}, d_{1}$ and $\lambda_{1}$ are also bounded.

When $d_{0}=0$ is obtained in step 1 , the algorithm stops. In fact, since $G(x) \prec 0$, $G(x) \circledast I \prec 0$ then the solution of $(26)$ is $\lambda_{0}=0$. Thus, $\nabla f(x)=0$ and we have that $x$ is a KKT point associated with a null Lagrangian multiplier matrix.

In what follows we consider the case where, at every iteration, $d_{0} \neq 0$.

Lemma 9 The direction $d_{0}$ computed by the algorithm satisfies

$$
d_{0}^{\top} \nabla f(x) \leqslant-d_{0}^{\top} B d_{0} .
$$

Proof Multiplying the first equation of (17) by $d_{0}^{\top}$,

$$
d_{0}^{\top} \nabla f(x)=-d_{0}^{\top} B d_{0}-d_{0}^{\top} \nabla G(x) \lambda_{0} .
$$

In view of the second equation of (17),

$$
-d_{0}^{\top} \nabla G(x) \lambda_{0}=\lambda_{0}^{\top}(G(x) \circledast I)(\Lambda \circledast I)^{-1} \lambda_{0} .
$$


Then,

$$
d_{0}^{\top} \nabla f(x)=-d_{0}^{\top} B d_{0}+\lambda_{0}^{\top}(G(x) \circledast I)(\Lambda \circledast I)^{-1} \lambda_{0} .
$$

Thanks to assumption 5 and $7,(\Lambda \circledast I)^{-1}(G(x) \circledast I) \in \mathbb{S}_{-}^{m}$ and $B \in \mathbb{S}_{++}^{n}$ then,

$$
d_{0}^{\top} \nabla f(x) \leqslant-d_{0}^{\top} B d_{0} .
$$

As a consequence, if the direction $d_{0} \neq 0$, it is a descent direction of $f$ at $x$

Lemma 10 The search direction d computed by the algorithm satisfies

$$
d^{\top} \nabla f(x) \leqslant \xi d_{0}^{\top} \nabla f(x) .
$$

Proof In view of (30),

$$
d^{\top} \nabla f(x)=d_{0}^{\top} \nabla f(x)+\rho d_{1}^{\top} \nabla f(x) .
$$

If $d_{1}^{\top} \nabla f(x)>0$, using $(28)$,

$$
\rho d_{1}^{\top} \nabla f(x) \leqslant(\xi-1) d_{0}^{\top} \nabla f(x) .
$$

If $d_{1}^{\top} \nabla f(x) \leqslant 0$, using (29),

$$
\rho \leqslant \varphi\left\|d_{0}\right\|^{2} .
$$

Both cases verifies (46) with $\xi \in(0,1)$.

Since $d_{0}$ is a descent direction of $f$ at $x$, then $d_{0}^{\top} \nabla f(x)<0$. Lemma 10 implies that $d$ is also a descent direction of $f$ at $x$.

Lemma 11 The search direction $d$ and the parameter $\rho$ computed by the algorithm verifies:

$$
\varphi_{0}\left\|d_{0}\right\|^{2} \leqslant \rho \leqslant \varphi\left\|d_{0}\right\|^{2}
$$

and

$$
\|d\| \leqslant \delta\left\|d_{0}\right\|
$$

for some $\delta \geqslant 1$ and $\varphi_{0}>0$.

Proof Looking at (28) and (29), we see that $\rho \leqslant \varphi\left\|d_{0}\right\|^{2}$. By lemma 9 and assumption 7 ,

$$
-d_{0}^{\top} \nabla f(x) \geqslant \sigma_{1}\left\|d_{0}\right\|^{2} \text {. }
$$

If $d_{1}^{\top} \nabla f(x)>0$ and in view of (28) we have,

$$
\min \left\{\varphi, \frac{(1-\xi) \sigma_{1}}{d_{1}^{\top} \nabla f(x)}\right\}\left\|d_{0}\right\|^{2} \leqslant \rho .
$$

Since $d_{1}$ is bounded and (29), there exist $\varphi_{0}>0$ such that

$$
\varphi_{0}\left\|d_{0}\right\|^{2} \leqslant \rho \text {. }
$$

and then (47) is proved.

Now using (24) and the triangular property,

$$
\|d\|=\left\|d_{0}+\rho d_{1}\right\| \leqslant\left\|d_{0}\right\|+\rho\left\|d_{1}\right\|
$$

and using condition (47),

$$
\|d\| \leqslant\left\|d_{0}\right\|+\varphi\left\|d_{0}\right\|^{2}\left\|d_{1}\right\|=\delta\left\|d_{0}\right\|,
$$

where $\delta=1+\varphi\left\|d_{0}\right\|\left\|d_{1}\right\| \geqslant 1$. 
As a consequence of (47) and (48), $\rho$ and $\|d\|^{2}$ has the same order of magnitude, in particular,

$$
\varphi_{0}\|d(x)\|^{2} \leqslant \rho(x) \leqslant \varphi\|d(x)\|^{2}, \quad x \in \Omega_{a} .
$$

Lemma 12 It follows from assumption (3) that there exists a positive real number $L$ such that

$$
G(y) \preccurlyeq G(x)+D G(x)(y-x)+L\|y-x\|^{2} I
$$

where $x, y \in \Omega$.

Proof Since $G$ is $C^{1}$ we can apply the Mean Value Theorem [26]

$$
G(y)=G(x)+\sum_{i=1}^{n}\left(y_{i}-x_{i}\right) \frac{\partial G}{\partial x_{i}}(x+\xi(y-x))
$$

for some $\xi \in(0,1)$. We have also that

$$
\begin{aligned}
\left(y_{i}-x_{i}\right) & \frac{\partial G}{\partial x_{i}}(x+\xi(y-x)) \preccurlyeq\left(y_{i}-x_{i}\right) \frac{\partial G}{\partial x_{i}}(x) \\
+ & \left\|\frac{\partial G}{\partial x_{i}}(x+\xi(y-x))-\frac{\partial G}{\partial x_{i}}(x)\right\|\|y-x\| I
\end{aligned}
$$

Since $\frac{\partial G}{\partial x_{i}}$ verify the Lipschitz condition, there exist $L_{i}>0$ such that

$$
\left\|\frac{\partial G}{\partial x_{i}}(x+\xi(y-x))-\frac{\partial G}{\partial x_{i}}(x)\right\| \leqslant L_{i}\|\xi(y-x)\|=L_{i} \xi\|y-x\| .
$$

Then, using (53), (52) and (51), we have,

$$
G(y) \preccurlyeq G(x)+\sum_{i=1}^{n}\left(y_{i}-x_{i}\right) \frac{\partial G}{\partial x_{i}}(x)+L\|y-x\|^{2} I
$$

where $L=\xi \sum_{i=1}^{n} L_{i}$.

Due to assumption (1) the sequence $\left\{x^{k}\right\}_{k \in \mathbb{N}} \in \operatorname{int}\left(\Omega_{a}\right)$ generated by the algorithm have an accumulation point $x^{*} \in \Omega_{a}$. It follows that there exist $\mathbb{K}_{1} \subset \mathbb{N}$ such that $\left\{d\left(x^{k}\right), \rho\left(x^{k}\right), \bar{\Lambda}\left(x^{k}\right), G\left(x^{k}\right)\right\}_{k \in \mathbb{K}_{1}}$ converges to $\left\{d\left(x^{*}\right), \rho\left(x^{*}\right), \bar{\Lambda}\left(x^{*}\right), G\left(x^{*}\right)\right\}$.

In [19], was proved the existence of a lower bound $\tau_{f}>0$ such that at any $x \in \Omega_{a}$, condition (31) is verified for any $t \in\left[0, \tau_{f}\right]$.

Proposition 1 For all $x \in \Omega_{a}$ such that $\|d(x)\| \geq M$ there exist $\tau>0$ such that:

$$
G(x+t d(x)) \preccurlyeq 0
$$

for all $t \in[0, \tau]$. 
Proof Thanks to lemma 12, there exist $L>0$ such that

$$
G(x+t d(x)) \preccurlyeq G(x)+t D G(x) d+t^{2} L\|d(x)\|^{2} I
$$

Let $F(t, x)$ be the matrix in the right hand side of $(55)$, then,

$$
G(x+t d(x) \preccurlyeq F(t, x) .
$$

We are going to prove the existence of $\tau>0$ such that

$$
\operatorname{sym}(\Lambda F(t, x)) \preccurlyeq 0, \quad t \in[0, \tau],
$$

and since $\Lambda \succ 0$, lemma 3 tell us that,

$$
F(t, x) \preccurlyeq 0, \quad t \in[0, \tau],
$$

and then condition (54) is true.

Taking the symmetric part of $\Lambda F(t, x)$ and considering (21), we can write,

$$
\begin{aligned}
\operatorname{sym}(\Lambda F(t, x)) & =\operatorname{sym}((\Lambda-t \bar{\Lambda}) G(x))+ \\
& +t\left(t L\|d(x)\|^{2}-\rho(x)\right) \Lambda
\end{aligned}
$$

where $\bar{\Lambda}=\operatorname{smat}(\bar{\lambda})$ is given in $(25)$.

Since $\rho$ verifies (49), we have,

$$
\rho(x) \leqslant \varphi_{0}\|d(x)\|^{2} .
$$

Then for all $v$ such that $\|v\|=1$

$$
\begin{aligned}
v^{t}(\Lambda F(t, x)) v & \leq v^{t} \Lambda G(x) v \\
& -t\left(v^{t}(\bar{\Lambda} G(x)) v+\varphi_{0}\|d(x)\|^{2} \lambda^{I}\right)+ \\
& +t^{2} L\|d(x)\|^{2} \lambda^{S}
\end{aligned}
$$

The right hand side is non-positive when $t=0$ then, let us consider the following second order equation:

$$
\begin{gathered}
v^{t} \Lambda G(x) v-t\left(v^{t}(\bar{\Lambda} G(x)) v+\varphi_{0}\|d(x)\|^{2} \lambda^{I}\right)+ \\
+t^{2} L\|d(x)\|^{2} \lambda^{S}=0
\end{gathered}
$$

where $x \in \Omega_{a},\|d(x)\| \geq M$ and $v$ such that $\|v\|=1$.

Let $t(x, v)$ be the positive solution of $(62)$ :

$$
\begin{aligned}
t(x, v) & =\left(\frac{v^{t}(\bar{\Lambda} G(x)) v}{2 L\|d(x)\|^{2} \lambda^{S}}+\frac{\varphi_{0} \lambda^{I}}{2 L \lambda^{S}}\right)+ \\
& +\sqrt{\left(\frac{v^{t}(\bar{\Lambda} G(x)) v}{2 L\|d(x)\|^{2} \lambda^{S}}+\frac{\varphi_{0} \lambda^{I}}{2 L \lambda^{S}}\right)^{2}-\frac{v^{t} \Lambda G(x) v}{L\|d(x)\|^{2} \lambda^{S}}}
\end{aligned}
$$

Since $v^{t} \Lambda G(x) v \leq 0, \frac{\phi_{0} \lambda^{I}}{2 L \lambda^{S}}>0$ and considering lemma 5 it can be shown that $t(x, v)$ is positive in the compact

$$
\mathbb{S}=\Omega_{a} \cap\{x:\|d(x)\| \geq M\} \times\left\{v \in \mathbb{R}^{n}:\|v\|=1\right\},
$$

then there exist $\tau>0$ such that $G(x+t d(x)) \preccurlyeq 0$ for all $t \in[0, \tau]$. 
A consequence of proposition 1 is that if $x^{*} \in \Omega_{a}$, is an accumulation point of a sequence $\left\{x^{k}\right\}_{k \in \mathbb{N}}$ generated by the algorithm then $\left\|d\left(x^{*}\right)\right\|=0$.

Proposition 2 If $\left\|d\left(x^{*}\right)\right\|=0$ there exists $\delta>0$ and $\tau>0$ such that if $x^{k} \in$ $B\left(x^{*}, \delta\right) \cap \Omega_{a}$,

$$
G\left(x^{k}+t d\left(x^{k}\right)\right) \preccurlyeq 0
$$

for all $t \in[0, \tau]$.

Proof Since $\left\|d\left(x^{*}\right)\right\|=0$ thanks to the continuity of $d(x),\left\|d\left(x^{k}\right)\right\|$ goes to zero. It follows from (59) that if

$$
\max \left\{v^{\top}\left(\Lambda^{k}-t \bar{\Lambda}^{k}\right) G\left(x^{k}\right) v, \quad v \in \mathbb{R}^{m}, \quad\|v\|=1\right\} \leqslant 0 .
$$

then $(64)$ is verified.

Since $\Lambda^{k}$ and $G\left(x^{k}\right)$ commute and are symmetric real matrices then there exist an orthonormal matrix $P_{k}$ such that

$$
\begin{aligned}
\Lambda^{k} & =P_{k}^{t} D_{\lambda}^{k} P_{k} \\
G\left(x^{k}\right) & =P_{k}^{t} D_{g}^{k} P_{k}
\end{aligned}
$$

where $D_{\lambda}^{k}$ is the diagonal matrix of eigenvalues of $\Lambda^{k}$ and $D_{g}^{k}$ is the diagonal matrix of eigenvalues of $G\left(x^{k}\right)$.

Let $\overline{\bar{\Lambda}}^{k}=P_{k} \bar{\Lambda}^{k} P_{k}^{t}$ then we need to prove that there exist $\delta>0$ and $\tau>0$ such that if $x^{k} \in B\left(x^{*}, \delta\right) \cup \Omega_{a}$ we have

$$
v^{\top}\left(\left(D_{\lambda}^{k}-t \overline{\bar{\Lambda}}^{k}\right) D_{g}^{k}\right) v \leqslant 0 .
$$

for all $v \in \mathbb{R}^{m}, \quad\|v\|=1$.

Let $\overline{\bar{\Lambda}}^{k}=D_{\lambda}^{*}+M^{k}$, where $M^{k}=P_{k} \bar{\Lambda}^{k} P_{k}^{t}-D_{\lambda}^{*}$. Since $\overline{\bar{\Lambda}}^{k}$ converges to $D_{\lambda}^{*}$ for all $\epsilon>0$ there exist $\delta_{1}$ such that for all $x^{k} \in B\left(x^{*}, \delta_{1}\right) \cap \Omega_{a},\left|v^{\top}\left(M^{k} G\left(x^{k}\right)\right) v\right|<\epsilon$.

Since the eigenvalues of $D_{\lambda}^{k}$ are between $\lambda^{I}$ and $\lambda^{S}$ by hypothesis and the eigenvalues of $\Lambda^{*}$ are bounded, due to theorem of Weyl [20], there exist $\tau>0$ such that the eigenvalues of $D_{\lambda}^{k}-t D_{\lambda}^{*}$ are positive for all $t \in[0, \tau]$.

Then for a sufficiently small $\epsilon$ :

$$
v^{\top}\left(\left(D_{\lambda}^{k}-t D_{\lambda}^{*}\right) D_{g}^{k}\right) v-t v^{\top}\left(M^{k} G\left(x^{k}\right)\right) v \leqslant 0 .
$$

for all $v \in \mathbb{R}^{m}, \quad\|v\|=1$ and $t \in[0, \tau]$.

Theorem 2 Any accumulation point $x^{*}$ of the sequence $\left\{x^{k}\right\}$ generated by the algorithm is a stationary point of problem (1).

Proof Let $\left\{x^{k}\right\}_{k \in \mathbb{K}}$ with $\mathbb{K} \subset \mathbb{N}$ be a sequence converging to $x^{*}$.

Since $\Lambda^{k}, B^{k}, d^{k}$ and $\rho^{k}$ are bounded, there exist $\mathbb{K}_{1} \subset \mathbb{K}$ such that

$$
\left\{x^{k}, \Lambda^{k}, B^{k}, \rho^{k}, d^{k}\right\}_{k \in \mathbb{K}_{1}}
$$

converges to $x^{*}, \Lambda^{*}, B^{*}, \rho^{*}, d^{*}$.

The search direction is a continuous function of $x, A, B$ and $\rho$. Then, the sequence $\left\{d^{k}\right\}_{k \in \mathbb{K}_{1}}$ converges to $d^{*}$ and $d^{*}=d\left(x^{*}, \Lambda^{*}, B^{*}, \rho^{*}\right)$

Let $\left\{t^{k}\right\}_{k \in \mathbb{K}_{2}}$ where $\mathbb{K}_{2} \subset \mathbb{K}_{1}$ be a sequence converging to $t^{*}$. 
The limit $\left\|d\left(x^{*}\right)\right\|=0$. If not we suppose that $\left\|d^{*}\right\|>\eta_{d}>0$ and thanks to proposition 1 we conclude that $t^{*}>0$.

From the line search condition (31),

$$
f\left(x^{f o l(k)}\right) \leqslant f\left(x^{k}\right)+\eta \cdot t^{k} \cdot\left(d^{k}\right)^{\top} \nabla f\left(x^{k}\right) .
$$

where $f o l(k)$ is the element that follows $k$ in $\mathbb{K}_{2}$. Taking the limits for $k \rightarrow \infty$,

$$
f\left(x^{*}\right) \leqslant f\left(x^{*}\right)+\eta \cdot t^{*} \cdot\left(d^{*}\right)^{\top} \nabla f\left(x^{*}\right) .
$$

Then, $0 \leqslant\left(d^{*}\right)^{\top} \nabla f\left(x^{*}\right)$.

Using lemma 10 and taking the limit for $k \rightarrow \infty$,

$$
\left(d^{*}\right)^{\top} \nabla f\left(x^{*}\right) \leqslant \xi\left(d_{0}^{*}\right)^{\top} \nabla f\left(x^{*}\right) .
$$

Using lemma 9 and taking the limit for $k \rightarrow \infty$,

$$
\left(d_{0}^{*}\right)^{\top} \nabla f\left(x^{*}\right) \leqslant-\left(d_{0}^{*}\right)^{\top} B^{*} d_{0}^{*}
$$

Since $B^{*}$ is positive definite, $d_{0}^{*}=d^{*}=0$. But this is contradictory with $\left\|d^{*}\right\|>$ $\eta_{d}>0$, then, the unique possibility is $d^{*}=0$.

Considering equation (17), we have that $\left(x^{*}, \lambda_{0}^{*}\right)$ verifies the following equations:

$$
\begin{gathered}
\nabla f\left(x^{*}\right)+\nabla G\left(x^{*}\right) \lambda_{0}^{*}=0 \\
\left(G\left(x^{*}\right) \circledast I\right) \lambda_{0}^{*}=0 .
\end{gathered}
$$

Equation (70) is the first equation of (2). It remains to proof the complementary condition $G\left(x^{*}\right) \Lambda_{0}^{*}=0$.

If $x^{*} \in \operatorname{int}\left(\Omega_{a}\right)$, then $G\left(x^{*}\right) \prec 0$. Consequently $G\left(x^{*}\right) \circledast I$ is non singular and the linear system (71) has the unique solution $\lambda_{0}^{*}=0$. Then, $\Lambda_{0}^{*}=\operatorname{smat}\left(\lambda_{0}^{*}\right)=0$ and the complementarity condition holds.

Now consider the case when $x^{*} \notin \operatorname{int}\left(\Omega_{a}\right)$. We must prove that $G\left(x^{*}\right) \Lambda_{0}^{*}=$ 0 . Since equation $(71), \operatorname{sym}\left(G\left(x^{*}\right) \Lambda_{0}^{*}\right)=0$. To finish the proof, we show that $\operatorname{skw}\left(G\left(x^{*}\right) \Lambda_{0}^{*}\right)=0$ or, equivalently, that all eigenvalues of $G\left(x^{*}\right) \Lambda_{0}^{*}$ are real.

Let $k \in \mathbb{K}_{2}$. Firstly, the matrix $\Lambda_{0}^{k}$ is symmetric. Secondly, the point $x^{k}$ is in the interior of $\Omega_{a}$ then, $G\left(x^{k}\right) \prec 0$. In view of lemma $4, G\left(x^{k}\right) \Lambda_{0}^{k}$ has real eigenvalues. On the other hand, the sequence $\left\{G\left(x^{k}\right) \Lambda_{0}^{k}\right\}_{k \in K_{2}}$ converges to $G\left(x^{*}\right) \Lambda_{0}^{*}$. Since the eigenvalues are continuous functions, we conclude that $G\left(x^{*}\right) \Lambda_{0}^{*}$ has real eigenvalues.

The last result proves that the presented algorithm globally converges to the solution of the problem in the convex case. 


\section{Numerical Implementation}

In this section we include implementation details of the introduced algorithm.

We code the algorithm of section 2.1 in a MATLAB function. The parameters are fixed with the following values: $\xi=0.8, \eta=0.1, \varphi=1, \nu=0.7$. Those values are commonly used in the "Feasible Direction Interior Point Algorithm" (FDIPA) [19]. Matrix $B$ and $\Lambda$ are initialized with the identity matrix.

Initially, the present algorithm takes as input, an interior point $x$. In order to mount the system matrix of (26), we compute $f(x), G(x), \nabla f(x), \nabla G(x)$, $\Lambda \circledast I$ and $I \circledast G(x)$. The symmetric Kronecker products are computed with the help of a MATLAB function used in SDPPACK [5]. Our actual implementation is appropriated for $G(x)$ of limited size. This limitation comes from the fact that we do not explore the sparsity of $G(x)$ and the size of the system matrix is $n+$ $\frac{1}{2} m(m+1)$.

Since the linear systems (26) and (27) are the same, we performs a single factorization per iteration of the algorithm.

Solving (26) we obtain $d_{0}(x) \in \mathbb{R}^{n}$ and $\Lambda_{0}(x) \in \mathbb{S}^{m}$. Let $T O L$ a positive constant. The optimization process stops if $\left\|d_{0}\right\|<T O L$ is verified. For small $T O L$, the condition $\left\|d_{0}\right\|<T O L$ means, in view of (17) and (18), that $x$ is near to a stationary point.

In order to accept the step length $t$ in Armijo's line search, we compute the maximum eigenvalue of $G(x+t d)$.

In step 3 matrix $B$ must be taken to verify assumption 7 . We use the BroydenFletcher-Goldfarb-Shanno (BFGS) updating rule with Powell's correction to ensure positive definiteness of $B$ [30].

On the other hand matrix $\Lambda$ must satisfy assumptions 5 and 6 . In order to verify assumptions 6 , we use the following " $\Lambda_{0}$-update" rule:

1. Compute $\lambda_{0 \min }$, the minimum eigenvalue of $\Lambda_{0}$.

2. If $\lambda_{0 \text { min }} \geqslant \lambda^{I}$ then, set $\Lambda=\Lambda_{0}$.

3. Else, set $\Lambda=\Lambda_{0}+\left(\lambda^{I}-\lambda_{0 \text { min }}\right) I$.

When $\Lambda_{0}$ is not positive definite, the effect of this rule is to obtain a matrix $\Lambda$ with all the eigenvalues of $\Lambda_{0}$ shifted to the right such that the minimum eigenvalue of $\Lambda$ be equal to $\lambda^{I}$. Since $\Lambda_{0}$ is bounded, this rule generates $\Lambda$ verifying assumption 6 . Unfortunately, when $x$ is not a stationary point, $\Lambda_{0}$ do not commute with $G(x)$. Then, $\Lambda$ generated with the $\Lambda_{0}-$ update rule do not commutes with $G(x)$. However, in practice, using this approach, the system matrix (26) have a bounded condition number.

\section{Applications in Structural Optimization}

In this section an overview of three semidefinite models of truss topology optimization are presented: the minimum compliance problem, the robust truss topology design and the problem of maximizing the minimal eigenvalue. We present numerical examples of those models in section 6. 
5.1 Minimum compliance problem

Consider a two or a three-dimensional truss with $b$ bars. Let $l$ be the degrees of freedom of the truss. The design variables of the our problems are the volumes of the bars denoted by $x_{j}, j=1, \ldots, b$. We define the worst possible compliance of the structure for the set of loadings $S \subset \mathbb{R}^{l}$ as,

$$
\phi_{S}(x)=\sup \left\{2 u^{\top} p-u^{\top} K(x) u: u \in \mathbb{R}^{l}, p \in S\right\},
$$

where $K(x)$ is the reduced stiffness matrix.

A typical model is the minimum compliance problem, also called Truss Topology Design (TTD) [9]. This problem considers structures submitted to a finite set of nodal loads called "primary" loads, and looks for the volume of each bar that minimizes the structural compliance. The structural topology changes if the volume of some of the bars are zero in the solution. Let consider the set of primary load cases as,

$$
P=\left\{p^{1}, \ldots, p^{s}\right\}
$$

where $p^{i} \in \mathbb{R}^{l}$ is the $i$-th load case. We can write the problem of minimum compliance as:

$$
\begin{array}{ll}
\min _{x, \tau} \tau & \\
\text { s.t. } & \phi_{P}(x) \leqslant \tau, \\
& \sum_{j=1}^{b} x_{j} \leqslant \bar{V}, \\
& x \geqslant 0,
\end{array}
$$

where $\bar{V}$ is a prescribed value for the total volume of the truss. Using the following equivalence proved in [6],

$$
\phi_{P}(x) \leqslant \tau \Longleftrightarrow\left[\begin{array}{cc}
\tau & p^{\top} \\
p & K(x)
\end{array}\right] \succcurlyeq 0, \quad p \in P,
$$

it is possible to rewrite (74) as the following semidefinite program:

$$
(T T D) \begin{cases}\min _{x, \tau} \tau & \\
\text { s.t. } \quad & {\left[\begin{array}{ll}
\tau & p^{\top} \\
p K(x)
\end{array}\right] \succcurlyeq 0, \quad p \in P,} \\
& \sum_{j=1}^{b} x_{j} \leqslant \bar{V}, \\
& x \geqslant 0 .\end{cases}
$$

\subsection{Robust truss topology design}

The following development was proposed by Ben-Tal and Nemirovsky [7]. In the robust truss topology problem the structure is "robust" if it is reasonable rigid when any set of small possible uncertain loads act on it. In additional to the primary loads, we includes a set of "secondary" loads that are uncertain in size and direction and can act over the structure. The compliance to be minimized is the worst possible one, considering "primary" and "secondary" load cases.

Let $M$ be the ellipsoid of loading conditions defined as follows:

$$
M=\left\{Q e: e \in \mathbb{R}^{q}, e^{\top} e \leqslant 1\right\},
$$


where

$$
Q=\left[\begin{array}{llll}
p^{1} & \ldots & s & p^{1} \\
& \ldots r e^{q-s}
\end{array}\right] \in \mathbb{R}^{l \times q} .
$$

The vectors $r e^{1}, \ldots, r e^{q-s}$ are called "secondary" load cases. The set $\left\{e^{1}, \ldots, e^{q-s}\right\}$ must be chosen as an orthonormal basis of a linear subspace orthogonal to the linear span of $P$. The value $r$ is a prescribed magnitude.

The robust truss topology problem $(T T D)$ is obtained replacing $P$ by $M$ in (74). In a similar way, using an equivalence similar to (75) proved in [7], it is possible to rewrite the robust truss topology model as the following semidefinite programming problem:

$$
(R T T) \begin{cases}\min _{x, \tau} \tau & \\
\text { s.t. } & {\left[\begin{array}{cc}
\tau I & Q^{\top} \\
Q & K(x)
\end{array}\right] \succeq 0} \\
& \sum_{j=1}^{b} x_{j} \leqslant \bar{V} \\
& x \geqslant 0\end{cases}
$$

where $Q$ is defined in (78).

\subsection{Structural topology optimization with eigenvalues}

This subsection uses theorical results and semidefinite programming problems presented in [1] and [2].

The eigenvalues of a truss are governed by the following equation:

$$
K(x) v=\lambda M(x) v
$$

where $M(x)$ is the reduced mass matrix of the structure and $(\lambda, v) \in \mathbb{R} \times \mathbb{R}^{m}$ is an eigenvector-eigenvalue pair. The well-defined smallest eigenvalue of problem (80), as explained in [1] and [2], is:

$$
\begin{aligned}
& \lambda_{\min }: X \rightarrow \mathbb{R} \cup\{+\infty\} \\
& \lambda_{\min }(x)=\sup \{\lambda: K(x)-\lambda M(x) \succcurlyeq 0\}
\end{aligned}
$$

where $X=\left\{x \in \mathbb{R}^{b}: x \geqslant 0, x \neq 0\right\}$.

The constraint $\lambda_{\min }(x) \geqslant \lambda$ is equivalent to the semidefinite constraint $K(x)-$ $\lambda M(x) \succcurlyeq 0$, see [1]. Then, the topology optimization problem of minimum volume subject to constraints in the minimum eigenvalue and compliance is

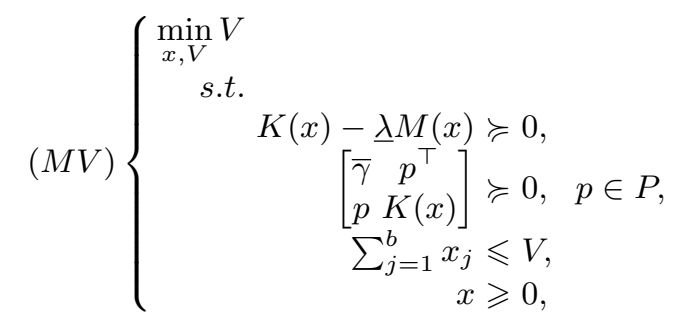

where $\underline{\lambda}$ is a lower bound to the minimum eigenvalue and $\bar{\gamma}$ an upper bound to the compliance. 
In a similar way, the problem of minimum compliance subject to constraints in the minimum eigenvalue and volume is

$$
(M C)\left\{\begin{aligned}
& \min _{x, \gamma} \gamma \\
& \text { s.t. } \\
& K(x)-\underline{\lambda} M(x) \succcurlyeq 0, \\
& {\left[\begin{array}{ll}
\gamma & p^{\top} \\
p & K(x)
\end{array}\right] } \succcurlyeq 0, \quad p \in P, \\
& \sum_{j=1}^{b} x_{j} \leqslant \bar{V}, \\
& x \geqslant 0,
\end{aligned}\right.
$$

where $\bar{V}$ is an upper bound to the volume of the structure.

Finally, the problem of maximizing the minimum eigenvalue with compliance and volume constraints is

$$
(M F)\left\{\begin{aligned}
& \max _{x, \lambda} \lambda \\
& \text { s.t. } \\
& K(x)-\lambda M(x) \succcurlyeq 0, \\
& {\left[\begin{array}{ll}
\bar{\gamma} & p^{\top} \\
p & K(x)
\end{array}\right] } \succcurlyeq 0, \quad p \in P, \\
& \sum_{j=1}^{b} x_{j} \leqslant \bar{V}, \\
& x \geqslant 0 .
\end{aligned}\right.
$$

In this paper we solve $(M F),(M V)$ and $(M C)$ and show its interrelation as in the example 4.1 of [1].

Problems $(T T D),(R T T),(M V)$ and $(M C)$ are convex optimization problems since the matrix constraint depends affinely on the design variables. On the other hand, problem $(M F)$ is non convex since the constraint $K(x)-\lambda M(x) \succcurlyeq 0$ depends linearly on the both design variables $x$ and $\lambda$.

\section{Numerical examples}

Examples 1 and 2 are applications of the present algorithm to problems (TTD) and $(R T T)$, respectively. In example 3 we apply the present algorithm to solve the problems $(M V),(M C)$ and $(M F)$. The structures of examples 1 and 2 are taken from [7] and the example 3 is from [1]. In all numerical tests, the optimization process stops when $\left\|d_{0}\right\|<10^{-6}$.

Recall that all problems solved here are first converted to the general format (1). Then, $n$ is the dimension of $x$ and represents the number of design variables and $m \times m$ is the size of $G(x)$ the constraint matrix.

Example 1. Consider the ground structure as shown in figure 1. In this example a single "primary" load $P=\{p\}$ is considered and the magnitude of each component of the load $p$ is 2.0. The length of each of the horizontal and vertical bars is 1.0. The secondary loadings have a magnitude $r=0.4$ and define a basis of the orthogonal complement of the linear span of $P$ in the linear space of all the degrees of freedoms of the structure.

Figure 2-left shows the final topology obtained with model $(T T D)$. The resulting truss is unstable since, for example, arbitrarily small non-horizontal force 


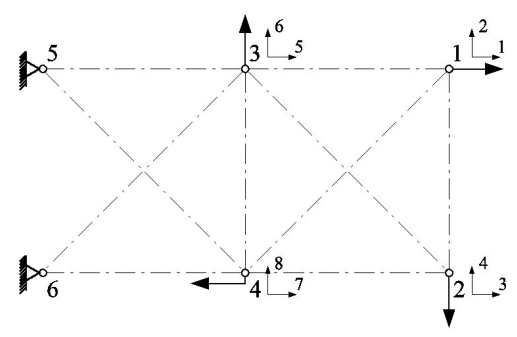

Fig. 1 Truss of Example 1.

applied to node 4 will produce infinite compliance. On the other hand, figure 2-right shows the final topology obtained with the robust model $(R T T)$.
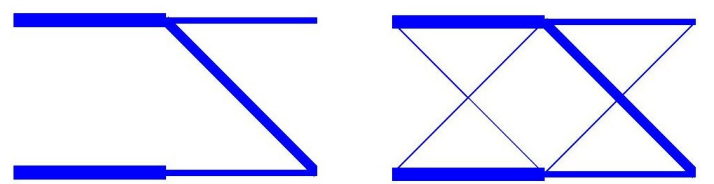

Fig. 2 Results of example 1. Left $=(T T D)$. Right $=(R T T)$.

Table 1 shows results obtained with the present algorithm. The row $n_{1}: n_{2}$ is the volume percentage of the bar connecting node $n_{1}$ and $n_{2}$. Vertical bars $3: 4$ and $1: 2$ are omitted since their volumes are null.

Table 1 Results of example 1. Bar volumes (\%).

\begin{tabular}{ccc} 
& $T T D$ & $R T T$ \\
\hline $3: 5$ & 25 & 24.482 \\
$1: 3$ & 12.5 & 11.954 \\
$4: 6$ & 25 & 24.483 \\
$2: 4$ & 12.5 & 11.954 \\
$4: 5$ & 0 & 1.2644 \\
$3: 6$ & 0 & 1.2644 \\
$2: 3$ & 25 & 23.679 \\
$1: 4$ & 0 & 0.9196 \\
\hline
\end{tabular}

The results presented in figure 2 and in table 1 are similar to the results shown in $[7]$.

Example 2. This example consists of a three-dimensional truss with fixed nodes on the horizontal plane $z=0$ and free nodes on the horizontal plane $z=2$. The 
structure has 8 nodes of coordinates,

$$
\begin{gathered}
{\left[\begin{array}{c}
\cos (2 \pi i / 4) \\
\sin (2 \pi i / 4) \\
0
\end{array}\right], i \in\{1,2,3,4\},} \\
{\left[\begin{array}{c}
\frac{1}{2} \cos (2 \pi j / 4) \\
\frac{1}{2} \sin (2 \pi j / 4) \\
2
\end{array}\right], j \in\{5,6,7,8\},}
\end{gathered}
$$

All the possible edges between free-free or free-fixed nodes are considered. A single load case $P=\{p\}$ is defined. The components of $p$ acting at the nodes on the plane $z=2$ are given by,

$$
p_{j}=\frac{1}{\sqrt{4\left(1+\rho^{2}\right)}}\left[\begin{array}{c}
\sin (2 \pi j / 4) \\
-\cos (2 \pi j / 4) \\
-\rho
\end{array}\right], \quad j \in\{5,6,7,8\}
$$

where $\rho=0.001$. The secondary loadings were built in the same way as in example 1 .

Figure 3-left and right shows the optimal result with model (TTD) and (RTT), respectively. The resulting structures of figure 3 differs only in the thickness of the "top" horizontal bars. The truss in figure 3-left has bars approximately 80 times smaller in volume than the right one. This result is also obtained in [7]
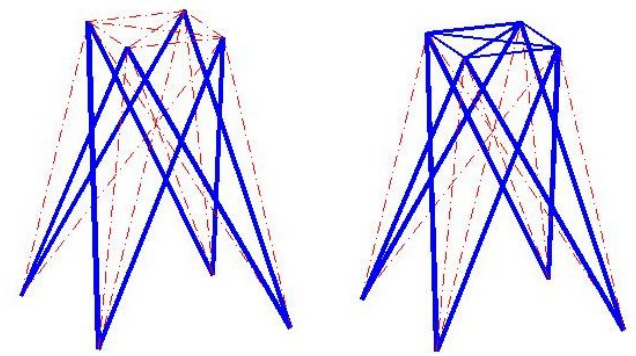

Fig. 3 Results of example 2. Left $=(T T D)$. Right $=(R T T)$.

Table 2 summarizes the executions of the present algorithm with example 1 and 2. The rows $n, m$ and iter are the number of design variables, the size of the constraint matrix $G(x)$ and the number of iterations, respectively. The resulting structure in both examples attains the maximum volume of $\bar{V}=1.0$.

Table 2 Results of example 1 and 2.

\begin{tabular}{ccccc} 
example & model & $n$ & $m$ & iter \\
\hline \multirow{2}{*}{1} & TTD & 11 & 20 & 22 \\
& RTT & 11 & 27 & 28 \\
\hline \multirow{2}{*}{2} & TTD & 23 & 36 & 19 \\
& RTT & 23 & 47 & 21 \\
\hline
\end{tabular}


Example 3.

Consider the planar structure with $3 \times 3$ nodes in figure 4 -left. The nodes in the left side are fixed in all directions and a horizontal force of magnitude 1.0 is applied in the middle node of the right side. The length of each of the horizontal and vertical bars is 1.0 and the Young's module of the material is 1.0. The initial structure has all bars with sectional area of 0.01 .

We consider the minimum volume problem $(M V)$ with $\bar{\gamma}=1$ and $\underline{\lambda}=0.05$. Our algorithm obtains the optimal design $\left(x^{*}, V^{*}\right)$ with $V^{*}=4.73$. The optimal design $x^{*}$ shown in figure 4-right. Since the optimal structure found is symmetric, only four components of $x^{*}$ are non-null and different each other. Those components are $1.58318,1.48329,1.57589 \times 10^{-1}$ and $6.75687 \times 10^{-1}$.

Considering the minimum compliance problem $(M C)$ with $\bar{V}=V^{*}=4.731$ and $\underline{\lambda}=0.05$, we obtain the optimal design $\left(x^{*}, \gamma^{*}\right)$ with optimal compliance $\gamma^{*}=\bar{\gamma}=1$.

Finally, when solving the problem of maximization the minimum eigenvalue $(M F)$ with $\bar{V}=V^{*}=4.731$ and $\bar{\gamma}=1$, we obtain the optimal design $\left(x^{*}, \lambda^{*}\right)$ with $\lambda^{*}=\underline{\lambda}=0.05$.

This result is also obtained in example 4.1 of [1].
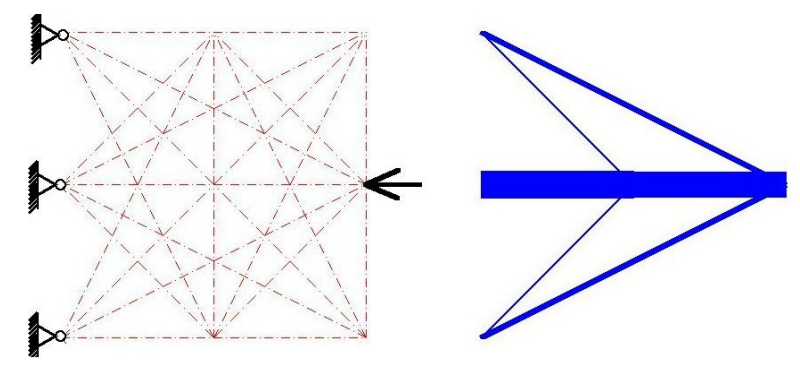

Fig. 4 Truss of example 3. Our algorithm obtains the same $x^{*}$ when applied to problems $(M V),(M C)$ and $(M F)$.

Test problems from SDPLIB and $C O M P l_{e} i b$

Here we present some results with test problems of $S D P L I B$ [11]. SDPLIB is a collection of linear semidefinite programming test problems. In this case the constraint matrix $G(x)$ is an affine combination of symmetric matrices and the objective function $f(x)$ is linear.

The test problems do not provide feasible points, then we first obtain a feasible point by solving the following auxiliary problem,

$$
\min _{x \in \mathbb{R}^{n}, z \in \mathbb{R}} z
$$

where $z$ is a new variable. Making iteration with our algorithm to solve (87), a feasible point $x_{0}$ is obtained once $z$ becomes negative. Then, $x_{0}$ is taken as the starting point to solve (1).

The results presented in table 3 , were solved by our algorithm using the same default parameters indicated in section 4 . The algorithm stops when $\left\|d_{0}\right\|<10^{-6}$. In column iter we write, iter $1+$ iter 2 , where iter 1 is the number of iterations to 
solve (87) to obtain feasible point $x_{0}$ and iter 2 is the number of iterations to solve problem (1) starting with $x_{0}$. Finally, in column $f$ is the value of the objective function obtained.

Table 3 Numerical results, $S D P L I B$.

\begin{tabular}{ccccc}
\hline Problem & $n$ & $m$ & iter & $f$ \\
\hline control 1 & 21 & 15 & $5+18$ & 17.7848 \\
control 2 & 66 & 30 & $6+30$ & 8.3001 \\
control 3 & 136 & 45 & $5+38$ & 13.6334 \\
control4 & 231 & 60 & $6+27$ & 19.7944 \\
hinf 1 & 13 & 14 & $6+39$ & 2.0326 \\
hinf 2 & 13 & 16 & $15+57$ & 10.9671 \\
hinf 3 & 13 & 16 & $22+39$ & 56.9425 \\
hinf 4 & 13 & 16 & $17+22$ & 274.766 \\
hinf5 & 13 & 16 & $25+52$ & 362.2963 \\
hinf6 & 13 & 16 & $18+37$ & 448.9428 \\
hinf 7 & 13 & 16 & $8+41$ & 390.8164 \\
hinf 8 & 13 & 16 & $14+405$ & 116.1638 \\
hinf 9 & 13 & 16 & $18+38$ & 236.2511 \\
hinf 10 & 21 & 18 & $12+41$ & 108.7538 \\
hinf 11 & 31 & 22 & $15+66$ & 65.899 \\
hinf 12 & 43 & 24 & $4+110$ & 2.0251 \\
hinf 14 & 73 & 34 & $18+137$ & 12.9927 \\
qap5 & 136 & 26 & $6+20$ & -435.9962 \\
qap6 & 229 & 37 & $3+207$ & -381.4346 \\
theta 1 & 104 & 50 & $3+12$ & 23.0002 \\
truss 1 & 6 & 13 & $4+15$ & -9 \\
truss 3 & 27 & 31 & $5+32$ & -9.1099 \\
truss 4 & 12 & 19 & $4+16$ & -9.0099 \\
\hline
\end{tabular}

Looking at table 3 , we can see that the numbers of iterations are comparable to the size of of the problem (except for hinf 8 , hinf 14 and qap6) and the value of the objective function is similar to the values published in [11].

\section{Conclusions}

In this paper, a new approach for nonlinear semidefinite programming is presented and supported by strong theoretical results. In particular, global convergence was proved for the convex case.

The present technique computes feasible and descent directions based on Newton-like iterations to solve KKT optimality conditions.

To obtain a search direction only two linear system with the same coefficient matrix are solved. The present technique is very robust and does not require tuning of parameters w.r.t. the considered application.

A set of numerical tests was performed with the same set of parameters.

Tests on non-convex problems show that the algorithm is still convergent. 


\section{Acknowledgments}

The authors thank the Brazilian Research Councils CAPES, CNPq, FAPERJ, the institutions supporting the program Ciência Sem Fronteiras of Brazil and the French Research Councils CNRS and INRIA for the financial support.

\section{References}

1. Achtzinger, A., Kočvara, M.: Structural topology optimization with eigenvalues. Tech. rep., University of Dortmunt (2006)

2. Achtzinger, W., Kočvara, M.: On the maximization of the fundamental eigenvalue in topology optimization. Structural and Multidisciplinary Optimization 34, 181-195 (2007)

3. Alizadeh, F.: Interior point methods in semidefinite programming with applications to combinatorial optimization. SIAM Journal on Optimization 5, 13-51 (1995)

4. Alizadeh, F., Haberly, J.P., Nayakkankuppam, M.V., Overton, M.L., Schmieta, S.: Sdppack user's guide - version 0.9 beta. Tech. rep., Courant Institute of Mathematical Science, NYU, New York, NY (1997)

5. Alizadeh, F., Haeberly, J.P.A., Nayakkankuppam, M.V., Overton, M.L., Schmieta, S.: Sdppack version 0.9 beta for matlab 5.0. http://cs.nyu.edu/overton/software/sdppack/sdppack.html (1997). URL http://cs.nyu.edu/overton/software/sdppack/sdppack.html

6. Ben-Tal, A., Nemirovski, A.: Potential reduction polynomial time method for truss topology design. Siam Journal of Optimization 4, 596-612 (1994)

7. Ben-Tal, A., Nemirovski, A.: Robust truss topology design via semidefinite programming. SIAM Journal on Optimization 7(4), 991-1016 (1997)

8. Ben-Tal, A., Nemirovski, A.S.: Lectures on Modern Convex Optimization: Analysis, Algorithms, and Engineering Applications. MPS/SIAM Series on Optimization (2001)

9. Bendsøe, M.P.: Optimization of structural topology, shape, and material. Springer-Verlag (1995)

10. Bonnans, F., Shapiro, A.: Perturbation Analysis of Optimization Problems. Springer Verlag (2000)

11. Borchers, B.: Sdplib 1.2, a library of semidefinite programming test problems. Optimization Methods and Software 11, 683-690 (1999)

12. Boyd, S., El Ghaoui, L., Feron, E., V., B.: Linear matrix inequalities in system and control theory. SIAM Studies in Applied Mathematics, SIAM, Philadelphia, USA (1994)

13. Correa, R., Ramirez, C.H.: A global algorithm for nonlinear semidefinite programming. SIAM Journal on Optimization 15, 303-318 (2004)

14. Fares B. Noll, D., Apkarian, P.: Robust control via sequential semidefinite programming. SIAM Journal on Control and Optimization 40, 17911820 (2002)

15. Fletcher, R.: A nonlinear programming problem in statistics (educational testing). SIAM Journal on Scientific Computing 2, 257267 (1981)

16. Goemans, M.X., Williamson, D.P.: Improved approximation algorithms for maximum cut and satisfiability problems using semidefinite programming. Journal of the ACM $\mathbf{7 9}$, 11151145 (1995)

17. Herskovits, J.: Developement of a numerical method for nonlinear optimization. Ph.D. thesis, Paris IX University, INRIA Rocquencort (1982)

18. Herskovits, J.: A two-stage feasible directions algorithm for nonlinear constrained optimization. Mathematical Programming 36, 19-38 (1986)

19. Herskovits, J.: A feasible directions interior point technique for nonlinear optimization. Journal of Optimization Theory and Applications 99, 121-146 (1998)

20. Horn, R.A., Johnson, C.R.: Matrix Analysis. Cambridge University Press (1985)

21. Jarre, F.: An interior method for nonconvex semidefinite programs. Optimization and Engineering 1, 347-372 (2000)

22. Kanzow, C., Nagel, C., Kato, H., Fukushima, M.: Successive linearization methods for nonlinear semidefinite programs. Computational Optimization and Applications 31, 251$273(2005)$

23. de Klerk, E.: Aspects of Semidefinite Programming: Interior Point Algorithms and Selected Applications. Kluwer (2002) 
24. Kočvara, M., Stingl, M.: Free material optimization for stress constraints. Structural and Multidisciplinary Optimization 33, 323-335 (2007)

25. Lewis, A.S., Overton, M.L.: Eigenvalue optimization. Acta Numerica 5, 149190 (1996)

26. Lima, E.L.: Anlise Real, vol. 2, 3 edn. Coleo Matemtica Universitria (2007)

27. Luenberger, D.G., Ye, Y.: Linear and Nonlinear Programming, 3 edn. Springer (2008)

28. M. Fukuda M. Kojima, K.M., Nakata, K.: Exploiting sparsity in semidefinite programming via matrix completions i: General framework. SIAM Journal of Optimization 11, 647674 (2000)

29. Panier, E.R., Tits, A.L., Herskovits, J.: A qp-free, globally convergent, locally superlinearly convergent algorithm for inequality constrained optimization. SIAM Journal of Control and Optimization 26, 788-810 (1988)

30. Powell, M.J.D.: The Convergence of Variable Metric Methods for Nonlinearly Constrained Optimization Calculations, in Nonlinear Programming 3. Academic Press, London (1978)

31. Shapiro, A.: First and second order analysis of nonlinear semidefinite programs. Mathematical Programming 77, 301-320 (1994)

32. Shapiro, A.: On uniqueness of lagrange multipliers in optimization problems subject to cone constraints. SIAM Journal on Optimization 7, 508518 (1997)

33. Stingl, M.: On the solution of nonlinear semidefinite programs by augmented lagrangian methods. Ph.D. thesis, University of Erlangen-Nrnberg (2005)

34. STINGL, M., KOCVARA, M., LEUGERING, G.: A sequencial convex semidefinite programming algorithm with an application to multiple-load free material optimization. SIAM Journal on Optimization 2009, 130-155

35. Stingl, M., Kočvara, M., Leugering, G.: Research report 319: Free material optimization with fundamental eigenfrequency constraints. Tech. rep., Institute of Applied Mathematics, University of Erlangen (2007)

36. Todd, M.J.: Semidefinite optimization. Acta Numerica 10, 515-560 (2001)

37. Zowe, J., Kocvara, M., Bendsoe, M.P.: Free material optimization via mathematical programming. Mathematical Programming 79, 445-466 (1997)

38. Zowe, J., Kocvara, M., Bendsoe, M.P.: Free material optimization via mathematical programming. the multi-load case with contact conditions. SIAM,J. Optimization 9, 813-832 (1997) 